\title{
Nutrient Intakes of Canadian Children and Adolescents: Results from the Canadian Community Health Survey (CCHS) 2015 - Nutrition Public Use Microdata Files
}

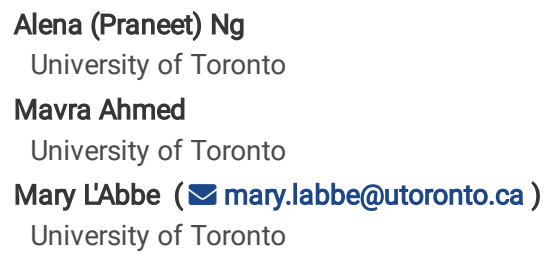

\section{Research Article}

Keywords: Nutrient intake, nutrient adequacy, nutrition surveillance, nutrition monitoring, children and adolescents, dietary assessment, Canadian Community Health Survey (CCHS), National Cancer Institute (NCl) method, Dietary Reference Intakes

Posted Date: December 22nd, 2021

DOI: https://doi.org/10.21203/rs.3.rs-1138934/v1

License: () (1) This work is licensed under a Creative Commons Attribution 4.0 International License. Read Full License 


\section{Abstract}

Background: Up-to-date and appropriate estimates of the usual intakes of key nutrients are crucial for monitoring the nutritional adequacy of the Canadian population. Comprehensive, nationally-representative nutrient estimates for Canadian children and adolescents are available using data from the Canadian Community Health Survey (CCHS) - Nutrition 2004, however results are scarce for the most-recent 2015 data. The objective of this research was to assess nutrient intakes of Canadian children and adolescents using data from the CCHS 2015 Public Use Microdata Files (PUMF)

Methods: Participants' first 24-hr dietary recall, and the second-day recall from a subset of participants were used to estimate usual intakes of macronutrients, vitamins and minerals in children and adolescents (2-18 years). Usual intakes by Dietary Reference Intake (DRI) age-sex groups were estimated using the National Cancer Institute ( $\mathrm{NCl}$ ) method, adjusted for age, sex, misreporting status, weekend/weekday, and sequence of recall analyzed (first/second), with consideration for outliers $(N=5,493)$. Usual intakes from food and beverages were assessed for prevalence of inadequacy in relation to the DRI recommendations.

Results: Children 2-3y consumed a percentage of total energy from protein above the Acceptable Macronutrient Distribution Range (AMDR). Among children and adolescents, $71 \%$ met the AMDR for carbohydrates and $63-71 \%$ met the AMDR for total fat. With the exception of calcium and vitamin D, a very low prevalence $(<10 \%)$ of inadequate intakes was observed for select micronutrients among Canadian children 2-8y, while a substantial proportion of adolescents $(>15 \%)$ did not meet requirements for vitamin $A$, vitamin $C$, vitamin $D$, calcium, magnesium and zinc.

Conclusions: Canadian children and adolescents may not be meeting recommendations for short fall nutrients such as calcium, potassium, vitamin $\mathrm{A}$ and vitamin D. Fibre intake continues to remain low, while sodium intake exceeded recommendations. These findings provide important, updated baseline estimates on the nutrient intakes of this subpopulation in Canada for continued monitoring of adherence to the 2019 Canada's Food Guide and may be useful to inform future public health nutrition policies, programs and initiatives.

\section{Introduction}

Obesity rates for Canadian children and adolescents have nearly tripled in the last 30 years, with a reported $30 \%$ of children between the ages of 5 - 17 years overweight or obese $(1,2)$. Overweight and obesity increases the risk for non-communicable diseases (NCDs) such as cardiovascular diseases and diabetes, with these conditions persisting into adulthood $(3,4)$. Establishing healthy behaviors in childhood may mitigate some of the effects of NCDs in adulthood $(5-$ 7). One such behavior modification includes following a healthy eating pattern that includes nutrient-dense foods, to ensure that nutrient intakes are met, while supporting periods of growth and development, and to maintain a healthy body weight (8).

Evidence suggests that the majority of Canadian children and adolescents do not meet national dietary guidelines for vegetable, fruit or dairy intake, and that their overall diet quality is poor (9-11). Previous research from the 2004 on the nutritional quality of the diets of Canadian children and adolescents show an overall low score for the Canadian Healthy Eating Index (i.e. less than 50 out of a maximum score of 100 points) (12) due to low intakes of nutrient-dense foods (13). Although the proportion of young Canadians that consume a high-quality diet has increased from 2004 to 2015 , less than $30 \%$ of children and youth had high-quality diet scores (14). This sub-optimal diet quality is further compounded by children and adolescents' exposure, across multiple settings and via multiple modes of digital media, to discretionary foods marketing $(15,16)$.

Although there are several studies evaluating dietary intakes and related health outcomes among Canadian adults, studies pertaining to the diet quality of children and adolescents remains scarce. Previous research utilizing the 2004 cycle of the Canadian Community Health Survey (CCHS) - Nutrition, Canada's nationally-comprehensive health and nutrition survey, found that saturated fat and sodium intakes of both children and adolescents were higher than recommended, and $20 \%$ of children and $30 \%$ of adolescents consumed kilocalories in excess of their energy requirements $(17,18)$. Additionally, more than $10 \%$ of adolescents were not meeting the requirements for vitamin A and magnesium, and among young Canadian females, intakes of vitamin B6, vitamin B12, folate, iron, and zinc were low $(17,18)$.

Additional results released from the 2015 CCHS-Nutrition survey utilizing the one-day dietary recall indicated a decrease in the percentage of total energy intake from carbohydrates and an increase in the percentage of total energy intake from protein compared to 2004 CCHS-Nutrition among children and adolescents, with no change in Canadians' energy intake from fat (19). Additionally, $72 \%$ of the Canadian children between the ages of $4-13$ years old were found to consume too much sodium, with the consumption of excess sodium peaking during adolescence (20). To date, Health Canada has released results on intakes of macronutrients by Dietary Reference Intakes (DRI) age-sex groups and results for sodium using 2015 CCHS-Nutrition data (20, 21); however, these results were from analyses using only the first day of dietary recall data. Recently, Health Canada released an Excel spreadsheet of Canadians' micronutrient intakes and prevalence of inadequacy utilizing the CCHS Share Files (a subset of 2015 CCHS - Nutrition which is not available publicly) without accompanying interpretation of the results [22]. A comprehensive understanding of the nutritional health of Canadian children and adolescents is needed to provide insight into key nutrients that may be important for public health monitoring and policy interventions for this subpopulation, and will aid in nutrition surveillance for years to come.

The objectives of this study were to estimate the usual intakes of macronutrients and micronutrients of Canadian children and adolescents (2-18 years) by DRI age-sex groups and to compare these intakes to the DRIs (22-24) to assess the prevalence of nutrient inadequacy. SAS and R-Studio macros were developed to analyze the 2015 CCHS-Nutrition Public Use Microdata File (PUMF) (freely available and downloadable from Statistics Canada (25) for the intakes of all survey respondents. Coding was adapted to apply the National Cancer Institute (NCl) method (26), adjusted for misreporting bias and covariates when calculating nutrient inadequacies, correction for missing measured body weight, and outlier removal.

\section{Materials And Methods}




\section{Data Source and Study Population}

Data were from 2015 CCHS-Nutrition, which is a voluntary, nationally-representative health and nutrition survey that provides food and beverage intake data for Canadians from all provinces (27). For study analyses, the 2015 CCHS PUMF were used; access can be requested through Health Canada (25), with detailed information available online (28).

Briefly, a complex multi-clustered, cross-sectional design was used to randomly select one individual per household for survey completion (28). The sampling frame in 2015 CCHS was Canadians aged $\geq 1$ years of age residing in the provinces and excluded individuals living in the territories and on reserve, full-time members of the Canadian Armed Forces, and those living in prison or long-term care facilities (28). Two questionnaires were administered per household: 1 ) a general health questionnaire to collect demographic, health and lifestyle measures and anthropometric data, and 2) a 24-hour dietary recall to assess all food and beverage intake of an individual for the past 24-hours (28). Heights and weights were measured by trained interviewers in-home, in addition to the administration of the 24-hr dietary recall. A computerized Canadian modification of the United States Department of Agriculture (USDA) five-step Automated Multiple Pass Method was used for dietary intake data (29). Energy and nutrient content of all foods and beverages reported were derived from Health Canada's Canadian Nutrient File (CNF), version 2015 (30).

In 2015 CCHS, 20,487 respondents completed the initial 24-hr dietary recall and a random subsample of 7,608 completed the second recall by phone 3-10 days after; response rates were $61.6 \%$ and $68.6 \%$, respectively (28). For the purpose of this study, respondents were excluded if they were $>18$ years of age $(n=$ $13,919)$ or underweight $(n=1,075)$. The final analytical sample size was $n=5,493$. Applying the survey weights provided by Statistics Canada ensured all analyses were nationally-representative (28). Additional detailed information on 2015 CCHS - Nutrition can be found in the user guide (31).

\section{Handling misreporting of dietary intake}

To account for over- and under-reporting of energy intake, reported energy intakes (EI) in 2015 CCHS were compared to each respondents' total energy expenditure (TEE) based on the method proposed by Garriguet (32). Under- and over-reporting were defined as the ratio of El:TEE < 0.7 and $>1.42$, respectively; those in between were considered plausible reporters. TEE was calculated based on availability of age, sex, height, weight and physical activity levels (i.e. sedentary, low active, moderately active and highly active), as per the Institute of Medicine's energy expenditure equations (33). Body Mass Index (BMI) categories for children aged 18 years or younger were defined by Cole et al. (34) and were calculated using measured height and weight for the subsample of respondents who had these measurements. For children where measured height and weight were not available, USDA equations were used to estimate TEE; these equations require age, sex and physical activity level only (35). If physical activity levels were unavailable, children younger than 14 years of age were assumed to be low active while teenagers aged 14 years or older were assumed to be sedentary based on the cut-offs proposed by Garriguet (32).

\section{Assessing usual intakes and nutrient inadequacy}

Since the previous 2004 CCHS-Nutrition, new statistical methods for the analysis of usual dietary intakes have been proposed (36), with the NCI method recommended by Health Canada for the analysis of 2015 CCHS data $(26,36)$. Advantages of using the $\mathrm{NCl}$ method include the ability to adjust for covariates of interest in the estimation of usual intake, and more precise estimations of the usual intakes of episodically consumed foods (37, 38). In this study, the NCl method was applied using both days of 24-hr dietary recall where available to estimate all usual nutrient intakes across DRI age-sex groups (39).

Application of the $\mathrm{NCl}$ method to derive usual nutrient intakes, including considerations for the type of model to use (e.g. amount only or two-part), choice of covariates, stratification vs. pooling of the data by specific age-sex groups, and outlier removal were guided by recommendations from a joint Statistics Canada/Health Canada technical working group, detailed in the paper by Davis et al. $(28,36)$. For micronutrients, the amount-only univariate $\mathrm{NCl}$ macros were used (26). For percent of calories from macronutrients, the amount-only bivariate macros were used (26). The following covariates were adjusted for in all $\mathrm{NCl}$ models (with the exception of energy, where misreporting status was not adjusted for): age, sex, misreporting status, sequence of recall analyzed (first/second); and weekend/weekday recall, with Friday considered a weekend.

Outliers with implausible nutrient intakes were removed from all analyses as proposed by Davis et al (36). All means, percentiles and proportion of the population above or below a DRI cut-off were generated using the $\mathrm{NCl}$ method (26). Further details on the development and application of the NCI method can be found elsewhere $(37,38)$.

$\mathrm{NCl}$-derived usual nutrient intakes were assessed for nutrient adequacy by comparing the estimated intakes to the age- and sex-specific nutrient recommendations found in the DRIs $(23,33,40)$. Since DRIs have not been defined for saturated fat, intake of this nutrient was compared to the World Health Organization (WHO) cut-off of $10 \%$ of total energy (5). Based on the threshold proposed by Health Canada, low prevalence of inadequacy was defined in this study as population estimated intakes $<10 \%$ of the Estimated Average Requirement (EAR) $(41,42)$. In the case of iron for females of menstruating age (aged $14-18 y)$, the full probability approach was used to assess nutrient adequacy $(43,44)$, as the iron requirements distribution is known to be skewed for this age/sex group $(24,39,43,44)$.

\section{Statistical Analysis}

Analyses were performed using R-Studio (v1.1.447) and SAS version 9.4 (SAS Institute Inc., Cary, NC, USA). Data manipulation was conducted using R-Studio, while analysis was conducted using SAS. Bootstrap balanced repeated replication (BRR) with 500 replicates was used to estimate confidence intervals, standard errors and coefficients of variation due to the complex sampling design of 2015-CCHS. Survey weights provided by Statistics Canada were applied to all analyses to ensure nationally-representative estimates (28). Descriptive statistics based on estimated usual dietary intakes (e.g., means, standard errors and percentiles) and prevalence of inadequacy for nutrients were calculated and presented by nutrient for each age-sex group. Data manipulation and analytic codes, for 2015-CCHS PUMF, are available from the authors upon request. 


\section{Results}

All results presented are means \pm standard errors (SEs). For detailed distributions of estimated usual nutrient intakes (5th, 10th, 25th, 50th, 75th, 90th and 95th ) and comparison to the DRIs for each DRI age-sex group, see Supplementary Tables (S1-S27).

\section{Percentage of Energy from Macronutrients}

Mean percentages of total energy from carbohydrates, total fat and protein were $49 \%, 16 \%, 33 \%$, respectively (Table 1, Figure 1a/1b, Table S2-S4). With the exception of 2-3y, estimated mean percentages of total energy from protein were within the Acceptable Macronutrient Distribution Range (AMDR), while a smaller proportion of Canadian children and adolescents had energy intakes from carbohydrates ( 70\%) and total fat within the AMDR ( 64\%).

Table 1

Percentage of Total Energy from Macronutrients and Dietary Fats (\%). Usual intakes from food and beverages by DRI age-sex group, Canadian children ar removal ${ }^{1-3}$

\begin{tabular}{|c|c|c|c|c|c|c|c|c|c|c|c|c|c|c|c|c|c|c|c|}
\hline & \multicolumn{3}{|c|}{ Carbohydrates } & \multicolumn{3}{|c|}{ Protein } & \multicolumn{3}{|c|}{ Total Fat } & \multicolumn{3}{|c|}{ Saturated Fat } & \multicolumn{3}{|l|}{ PUFA } & \multicolumn{3}{|l|}{ MUFA } & \multirow{2}{*}{$\begin{array}{l}\text { Lino } \\
n\end{array}$} \\
\hline & $n$ & Mean & SE & $n^{4}$ & Mean & SE & $n$ & Mean & SE & $n$ & Mean & SE & $n$ & Mean & SE & $n$ & Mean & SE & \\
\hline \multicolumn{20}{|c|}{ Both sexes } \\
\hline $\begin{array}{l}2- \\
3 y\end{array}$ & 780 & 49.3 & 0.5 & 780 & 16.2 & 0.2 & 780 & 33.5 & 0.3 & 780 & 10.8 & 0.1 & 780 & 7.1 & 0.1 & 780 & 12.3 & 0.2 & 780 \\
\hline $\begin{array}{l}4- \\
8 y\end{array}$ & 1117 & 49.3 & 0.5 & 1117 & 16.2 & 0.2 & 1117 & 33.4 & 0.3 & 1117 & 10.8 & 0.1 & 1117 & 7.1 & 0.1 & 1117 & 12.3 & 0.2 & $111^{-}$ \\
\hline \multicolumn{20}{|c|}{ Males } \\
\hline $\begin{array}{l}9- \\
13 y\end{array}$ & 952 & 49.3 & 0.5 & 952 & 16.2 & 0.2 & 952 & 33.4 & 0.3 & 952 & 10.8 & 0.2 & 952 & 7.1 & 0.1 & 952 & 12.3 & 0.2 & 952 \\
\hline $\begin{array}{l}14- \\
18 y\end{array}$ & 850 & 49.3 & 0.5 & 850 & 16.2 & 0.2 & 851 & 33.4 & 0.3 & 851 & 10.8 & 0.1 & 851 & 7.1 & 0.1 & 851 & 12.3 & 0.2 & 851 \\
\hline \multicolumn{20}{|c|}{ Females } \\
\hline $\begin{array}{l}9- \\
13 y\end{array}$ & 890 & 49.3 & 0.5 & 890 & 16.2 & 0.2 & 890 & 33.4 & 0.3 & 890 & 10.8 & 0.2 & 890 & 7.1 & 0.1 & 890 & 12.3 & 0.2 & 890 \\
\hline $\begin{array}{l}14- \\
18 y\end{array}$ & 902 & 49.3 & 0.5 & 902 & 16.2 & 0.2 & 902 & 33.4 & 0.3 & 902 & 10.8 & 0.1 & 901 & 7.1 & 0.1 & 902 & 12.3 & 0.2 & 902 \\
\hline
\end{tabular}

Data Source: Statistics Canada, Canadian Community Health Survey, Nutrition (2015) - Public Use Microdata File. DRI: Dietary Reference Intakes. SE: StandaI

${ }^{1}$ All intakes are based on food and beverage consumption only and exclude intakes from supplements.

2 The National Cancer Institute method (NCI method) for estimating usual dietary intake was used. The following covariates were adjusted for in all NCl mod day of the week (weekend vs. weekday), and sequence of dietary recall analysed (first or second); dietary misreporting was not adjusted for to derive usual in were defined and removed using the methodology reported in Davis et al (36). The $\mathrm{NCl}$ methodology was applied to each nutrient by DRI age-sex grouping se between sample sizes and point estimates. The number of respondents removed due to outlier methodology varied between 1-5 respondents for each nutrien population-level estimates using sampling survey weights provided by Statistics Canada. All reported SEs were bootstrapped using the 500 boot weights pro'

${ }^{3}$ For detailed intake distributions and comparisons to the Acceptable Macronutrient Distribution Range (AMDR) for each nutrient, see Supplementary Tables

${ }^{4}$ Sample size after outliers were removed.

\section{Total Energy}

Mean estimated energy intakes for children $2-3 y$ was $1967 \pm 40 \mathrm{kcal} /$ day, similar to those $4-5 \mathrm{y}$ (1946 $\pm 26 \mathrm{kcal} /$ day). Male adolescents (9-13y and $14-18 \mathrm{y})$ had an estimated mean energy intake of $2193 \pm 25 \mathrm{kcal} /$ day and $2181 \pm 22 \mathrm{kcal} /$ day, respectively. Female adolescents (9-13y and $14-18 \mathrm{y})$ had an estimated total energy intake of $1702 \pm 37 \mathrm{kcal} /$ day and $1693 \pm 32 \mathrm{kcal} /$ day, respectively (Table 2 , Table S1). 
Table 2

Energy, Fibre, Cholesterol and Sodium Intake. Usual intakes from food and beverages by DRI age-sex group, Canadian children and adolescents, $n=5,493$ before outlier removal ${ }^{1-3}$

\begin{tabular}{|c|c|c|c|c|c|c|c|c|c|}
\hline & \multicolumn{3}{|c|}{ Energy (kcal/d) } & \multicolumn{3}{|c|}{ Fibre $(g / d)$} & \multicolumn{3}{|c|}{ Cholesterol (mg/d) } \\
\hline & $n^{4}$ & Mean & SE & $n$ & Mean & SE & $n$ & Mean & SE \\
\hline \multicolumn{10}{|c|}{ Both sexes (y) } \\
\hline $2-3$ & 780 & 1967 & 40 & 780 & 12.0 & 0.4 & 780 & 263 & 4 \\
\hline $4-8$ & 1117 & 1946 & 26 & 1117 & 15.6 & 0.4 & 1117 & 291 & 13 \\
\hline \multicolumn{10}{|c|}{ Males (y) } \\
\hline $9-13$ & 952 & 2193 & 25 & 952 & 17.0 & 0.4 & 952 & 280 & 7 \\
\hline $14-18$ & 851 & 2181 & 22 & 852 & 19.1 & 0.5 & 852 & 226 & 5 \\
\hline \multicolumn{10}{|c|}{ Females (y) } \\
\hline $9-13$ & 890 & 1702 & 37 & 890 & 15.1 & 0.4 & 890 & 311 & 9 \\
\hline $14-18$ & 902 & 1693 & 32 & 902 & 14.9 & 0.4 & 902 & 310 & 7 \\
\hline \multicolumn{10}{|c|}{$\begin{array}{l}\text { Data Source: Statistics Canada, Canadian Community Health Survey, Nutrition (2015) - Public Use Microdata File. DRI: Dietary Reference Intakes. SE: } \\
\text { Standard Error. }\end{array}$} \\
\hline \multicolumn{10}{|c|}{${ }^{1}$ All intakes are based on food and beverage consumption only and exclude intakes from supplements. } \\
\hline \multicolumn{10}{|c|}{$\begin{array}{l}2 \text { The National Cancer Institute method (NCI method) for estimating usual dietary intake was used. The following covariates were adjusted for in all NCl } \\
\text { models: age, sex, dietary misreporting status, day of the week (weekend vs. weekday), and sequence of dietary recall analysed (first or second); dietary } \\
\text { misreporting was not adjusted for to derive usual intake of energy. Outliers for nutrient intake were defined and removed using the methodology reported in } \\
\text { Davis et al ( } 36 \text { ). The NCl methodology was applied to each nutrient by DRI age-sex grouping separately, hence small discrepancies between sample sizes } \\
\text { and point estimates. The number of respondents removed due to outlier methodology varied between } 1-5 \text { respondents for each nutrient. All estimates were } \\
\text { weighted for population-level estimates using sampling survey weights provided by Statistics Canada. All reported SEs were bootstrapped using the } 500 \\
\text { boot weights provided by Statistics Canada. }\end{array}$} \\
\hline \multicolumn{10}{|c|}{${ }^{3}$ For detailed intake distributions and comparisons to the Dietary Reference Intakes (DRIs) for each nutrient, see Supplementary Tables (S1-S27). } \\
\hline${ }^{4} \mathrm{Sa}$ & ed. & & & & & & & & \\
\hline
\end{tabular}

Percentage of Energy from Saturated Fat, Monounsaturated Fat and Polyunsaturated Fat

The mean percentage of total energy from saturated fat was approximately $11 \%$, while their mean intake from monounsaturated and polyunsaturated fatty acids were $12 \%$ and $7 \%$, respectively (Table 1, Tables S5-S7). About 76\% of Canadian children and adolescents had linoleic acid (omega-6) and alpha-linolenic acid (omega-3) intakes within the AMDR, with a mean percent of total energy of $6 \%$ and $0.75 \%$, respectively (Table 1, Tables S8-S9). Cholesterol intake ranged from $263 \pm 4 \mathrm{mg} /$ day for 2-3y to $311 \pm 9 \mathrm{mg} / \mathrm{d}$ for females 9-13y (Table 2, Table S11).

\section{Intakes of Vitamins and Minerals with an EAR}

With the exception of calcium and vitamin D, a very low prevalence of inadequate intakes was observed among Canadian children 2-8y. Among both males and female children (2-3y), less than $5 \%$ of the sample had inadequate intakes of fat-soluble vitamins (A, C), B vitamins (B6, B12, riboflavin, thiamin, niacin and folate) and trace elements (magnesium, phosphorus, iron and zinc) and less than 10\% had inadequate intakes of calcium (Table 3, Tables S12-S26). Similarly, for children aged $4-8 y$, less than $5 \%$ of the sample had inadequate intakes of fat-soluble vitamins (A, C), B vitamins (B6, B12, riboflavin, thiamin, niacin and folate) and trace elements (magnesium, phosphorus, iron and zinc), whereas the prevalence of inadequate intake of calcium was high at $31 \%$ (Table 3, Tables S12-S26). Greater than $91 \%$ of children aged 2-8y had inadequate intakes of vitamin D (Table 3, Table S14). 
Table 3

Micronutrient Intake. Usual intakes from food and beverages by DRI age-sex group, Canadian children and adolescents, $\mathrm{n}=$

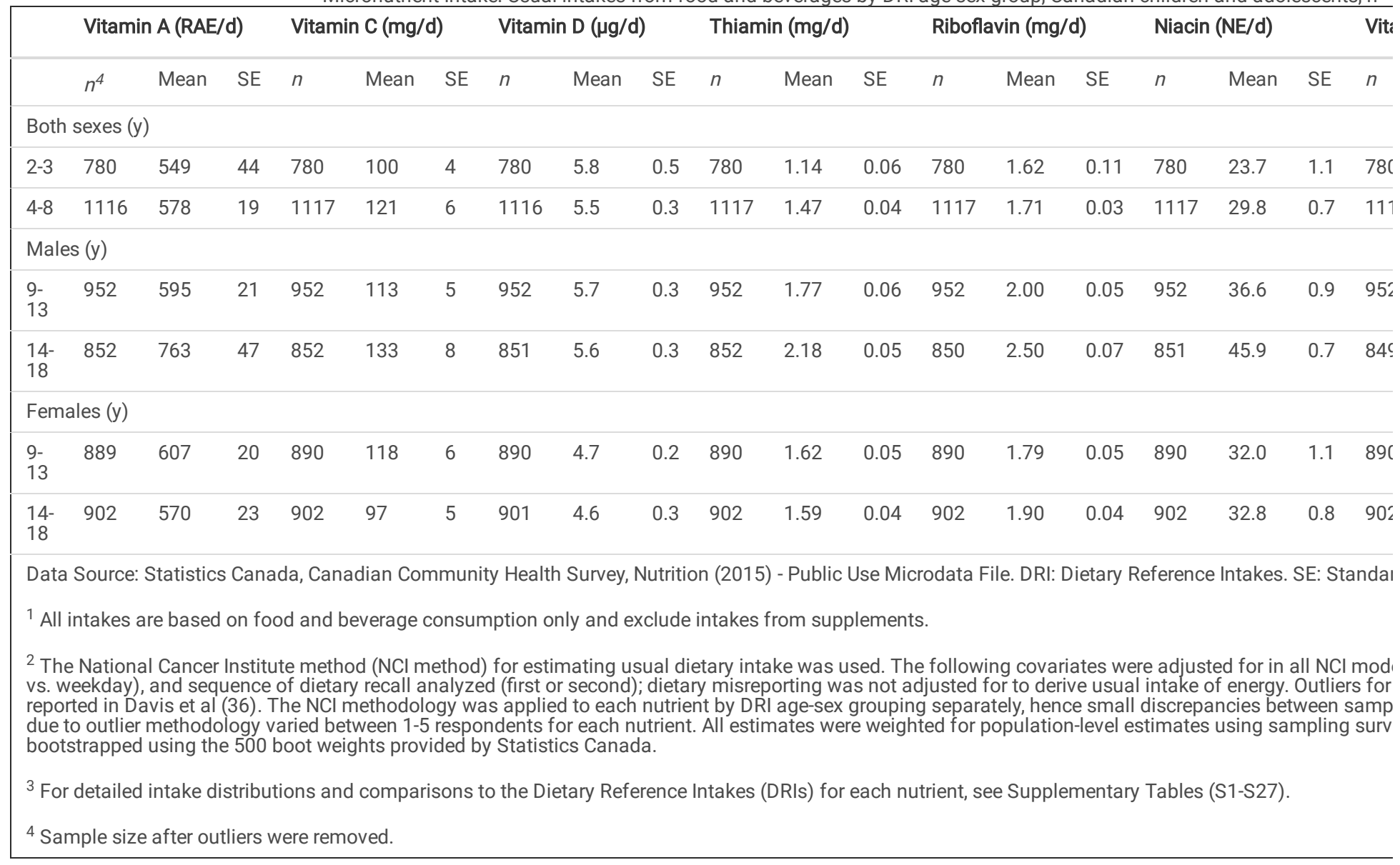


Table 3

Cont'd. Usual intakes from food and beverages by DRI age-sex group, Canadian children and adolescents, $n=5,493$ before outlier removal1-

\begin{tabular}{|c|c|c|c|c|c|c|c|c|c|c|c|c|c|c|c|c|c|c|c|}
\hline & \multicolumn{3}{|c|}{ Calcium (mg/d) } & \multicolumn{3}{|c|}{ Iron (mg/d) } & \multicolumn{3}{|c|}{ Magnesium (mg/d) } & \multicolumn{3}{|c|}{ Phosphorus (mg/d) } & \multicolumn{3}{|c|}{ Zinc (mg/d) } & \multicolumn{3}{|c|}{ Potassium (mg/d) } & \multirow[t]{2}{*}{$S$} \\
\hline & $n^{4}$ & Mean & SE & $n$ & Mean & SE & $n$ & Mean & SE & $n$ & Mean & SE & $n$ & Mean & SE & $n$ & Mean & SE & \\
\hline \multicolumn{20}{|l|}{$\begin{array}{l}\text { Both } \\
\text { Sexes } \\
\text { (y) }\end{array}$} \\
\hline $2-3$ & 780 & 933 & 64 & 780 & 8.2 & 0.4 & 780 & 211 & 6 & 780 & 1070 & 50 & 780 & 7.0 & 0.3 & 780 & 2056 & 92 & 7 \\
\hline $4-8$ & 1117 & 1028 & 28 & 1116 & 11.5 & 0.3 & 1117 & 241 & 4 & 1117 & 1169 & 21 & 1117 & 8.1 & 0.2 & 1117 & 2316 & 45 & 1 \\
\hline \multicolumn{20}{|l|}{$\begin{array}{l}\text { Males } \\
\text { (y) }\end{array}$} \\
\hline $9-13$ & 952 & 1058 & 36 & 952 & 13.8 & 0.4 & 952 & 272 & 7 & 952 & 1433 & 39 & 952 & 10.5 & 0.3 & 952 & 2552 & 56 & 9 \\
\hline $14-18$ & 852 & 1078 & 34 & 851 & 16.1 & 0.5 & 852 & 354 & 16 & 851 & 1572 & 40 & 852 & 13.4 & 0.6 & 851 & 3043 & 97 & 8 \\
\hline
\end{tabular}

Females

(y)

$\begin{array}{llllllllllllllllllll}9-13 & 890 & 882 & 22 & 888 & 11.9 & 0.3 & 890 & 247 & 6 & 890 & 1167 & 29 & 890 & 8.9 & 0.3 & 890 & 2248 & 45 & 8 \\ 14-18 & 902 & 888 & 26 & 902 & 11.3 & 0.3 & 902 & 256 & 7 & 902 & 1272 & 31 & 902 & 9.1 & 0.3 & 902 & 2300 & 49 & 9\end{array}$

Data Source: Statistics Canada, Canadian Community Health Survey, Nutrition (2015) - Public Use Microdata File. DRI: Dietary Reference Intakes. SE: StandaI

${ }^{1}$ All intakes are based on food and beverage consumption only and exclude intakes from supplements.

2 The National Cancer Institute method ( $\mathrm{NCl}$ method) for estimating usual dietary intake was used. The following covariates were adjusted for in all NCI mod dietary misreporting status, day of the week (weekend vs. weekday), and sequence of dietary recall analyzed (first or second); dietary misreporting was not ac derive usual intake of energy. Outliers for nutrient intake were defined and removed using the methodology reported in Davis et al (36). The NCI methodology each nutrient by DRI age-sex grouping separately, hence small discrepancies between sample sizes and point estimates. The number of respondents remove methodology varied between 1-5 respondents for each nutrient. All estimates were weighted for population-level estimates using sampling survey weights pre Canada. All reported SEs were bootstrapped using the 500 boot weights provided by Statistics Canada.

${ }^{3}$ For detailed intake distributions and comparisons to the Dietary Reference Intakes (DRIs) for each nutrient, see Supplementary Tables (S1-S27).

${ }^{4}$ Sample size after outliers were removed.

For adolescents (9-18y), there was a low prevalence of inadequate intakes (10-15\% below the EAR) for B vitamins (B6, B12, riboflavin, thiamin, niacin) (Table 3, Figure 2, Tables S15-S20). Although the prevalence of inadequacy for folate was low at $5 \%$ and $8 \%$ for males and females $9-13 y$, respectively, adolescents aged 14-18y had a high prevalence of inadequacy for this nutrient at $12 \%$ for males and $29 \%$ for females (Table 3, Figure 2, Table S19).

With respect to fat-soluble vitamins, the prevalence of inadequate intakes was high for fat soluble vitamins (vitamin $A$, vitamin $C$ and vitamin D). A substantial proportion of Canadian adolescents aged 9-18 y (males: 38-52\%; females: 18-41\%) consumed vitamin A in quantities below the EAR (Table 3, Figure 2, Table S12). Although the prevalence of inadequate vitamin $C$ intakes were low for adolescents aged $9-13 y,>16 \%$ of adolescents $14-18 y$ had inadequate intakes of this nutrient (Table 3, Figure 2, Table S13). Almost all Canadian adolescents $(>92 \%)$ had a high prevalence of inadequate intake of vitamin D (Table 3, Figure 2 , Table S14).

Inadequate intakes of trace elements were also observed for male and female adolescents. The prevalence of inadequacy for calcium ranged from $58-60 \%$ for male adolescents and 75-77\% for female adolescents (Table 3, Figure 2, Table S21), whereas for magnesium, prevalence of inadequate intakes were slightly lower at $16-53 \%$ for male adolescents and $25-75 \%$ for female adolescents; for phosphorus, $18 \%$ of male adolescents and $31-37 \%$ of female adolescents had inadequate intakes, respectively (Table 3, Figure 2, Table S23-S24). Although a low prevalence of iron inadequacy (<5\% below EAR) was found among adolescent males (9-18y) and females (9-13y), approximately 25\% of females 14-18y consumed iron in amounts that fell below their EAR (Table 3, Figure 2, Table S22). A substantial proportion of Canadian adolescents had inadequate intake of zinc, ranging from $15-21 \%$ for males and $23-28 \%$ for females (Table 3, Figure 2, Table S25).

With the exception of vitamin A and zinc among children aged 2-3y, the proportion of intakes from food and beverages that were greater than the Tolerable Upper Intake Level (UL) was very low for most vitamin and minerals.

Intakes of Vitamins and Minerals with an Adequate Intake (AI) or Chronic Disease Risk Reduction (CDRR) level

The majority of Canadian children and adolescents (> 93\%) had estimated fibre intakes below the Al for their respective age-sex groups (Table 2, Table S10).

The median potassium intakes of Canadian children and adolescents was below their respective Als (2300-3000 mg/day), and thus, no assessment can be made regarding the prevalence of inadequacy of potassium (Table 3, Table S26).

More than half $(66 \%)$ of the sample exceeded the CDRR for sodium. Males $14-18 \mathrm{y}$ had the highest estimated mean sodium intake at $3737 \pm 84 \mathrm{mg} / \mathrm{d}$, with $90 \%$ of this subpopulation above the CDRR recommendation (Table 2, Table S27). 


\section{Discussion}

This study presents results of analyses from the most-recent, nationally-representative health and nutrition survey of Canadians to estimate usual nutrient intakes of Canadian children and adolescents, and assesses the prevalence of nutrient inadequacy in relation to the DRIs. Results from this study showed that the majority of Canadian children and adolescents are meeting AMDR recommendations for macronutrients; however, many children may not be meeting requirements for vitamin $\mathrm{D}$ or calcium, while many adolescents may not be meeting requirements for vitamin $A$, vitamin $C$, vitamin $D$, calcium, magnesium and zinc. For nutrients with an Al, there is a concern that both children and adolescents may not be meeting their needs for potassium and fibre, while consumption of sodium was in excess of international recommendations.

Although the available literature on nutrient intakes of children and adolescents is limited, results from this study are consistent with those published on macronutrient intakes of Canadian children and adolescents using one day of dietary recall from the 2015 CCHS- Nutrition, is consistent with results on Canadian children and adolescents using the 2004 CCHS- Nutrition $(17,45)$, and is also consistent with results for US children and adolescents using the 2015-2016 National Health and Nutrition Examination Survey (NHANES) $(46,47)$. For example, inadequate intakes of vitamin A, vitamin D, magnesium and calcium is a concern for adolescents in both countries and within Canada between 2004 to 2015, and both children and adolescents may not be meeting their requirements for potassium and fibre $(17,18,45)$. Furthermore, the mean estimated energy intakes of Canadian children and adolescents reported in this study were similar to those found in previously published findings using 2004 CCHS and 2015 CCHS- Nutrition - the only exception being for 1-3y, where we observed a mean energy intake of $1,967 \mathrm{kcal} /$ day in our study compared to $1,585 \mathrm{kcal} /$ day in $2004-\mathrm{CCHS}$ and $1,308 \mathrm{kcal} / \mathrm{day}$ in $2015-\mathrm{CCHS}$ (48). However, this difference may be largely attributable to the exclusion of 1-year olds from our study (as they do not have a TEE equation), as well as methodological differences between this study and other published studies (48) (further discussed in Strengths and Limitations).

In this study, saturated fat contributed to $10 \%$ of total energy in the diets of children and adolescents. A high-energy, high-saturated fat diet and low activity lifestyle that contributes to obesity and NCDs in adults may be having the same impact on children and adolescents (8). The Canadian Health Measures Survey found that among Canadian children and adolescents, $7 \%$ had either borderline or elevated blood pressure measurements, a risk factor for cardiovascular disease (49). Highly processed foods are a major source of saturated fat in the Canadian diet and can also be high in calories, sodium and free sugars (50). Ultra-processed foods contribute more than $50 \%$ of total daily energy in the diets of Canadian children and adolescents (51). Considering the mounting evidence that increased consumption of ultra-processed foods has a negative impact on diet quality and health, continued surveillance on nutrient intakes and interventions (e.g., food policies and regulations addressing the food environment, marketing to kids etc.) to reduce consumption of unhealthy processed foods will remain a priority.

An assessment of Canadian children and adolescents' fibre intake with the recommendation is not possible given the limited usefulness of the Al in assessing nutrient adequacy of groups. Despite this limitation, the importance of an appropriate fibre intake should still be promoted to young Canadians, as recent reports indicate decreasing intakes of fruits, vegetables and whole grains, which are an important source of fibre and potassium (52).

Given its importance in the development of peak bone mass, low calcium intake is a significant health concern for children and adolescence and may play a role in the risk of fractures and developing osteoporosis later in life (53). Other than factors such as lactose intolerance or cultural/family practices and suboptimal intake of calcium may be linked to replacement of dairy products with sugar-sweetened beverages (46). Research has shown that soft-drink consumption is highest in adolescence while milk intake is lowest (54-56). In addition to calcium, milk is an important source of other shortfall nutrients such as vitamin D, phosphorus, magnesium, potassium, vitamin A, and zinc (8). Adolescent females are likely to drink less milk in order to cut kilocalories, favoring low-kilocalorie soft drinks (57).

Nearly $100 \%$ of Canadian children and adolescents had a high prevalence of inadequate intakes for vitamin D, similar to results from 2004-CCHS Nutrition (17, 45). This is a likely a reflection of a significant decrease in fluid milk consumption $(58,59)$, as all fluid milk in Canada is fortified with Vitamin $D$, and the change in DRI recommendations from an Al (5ug/day for children and adolescents) to a higher EAR (10ug/day) in 2011 (22). Estimates of the prevalence of inadequate intakes of vitamin D from food must be interpreted with caution as Vitamin D can also be synthesized by the body from sunlight (UV radiation) (60).. Additionally, this study does not consider vitamin D from supplements in the assessment; in 2004, 35\% of Canadian children and adolescents reported taking a nutritional supplement over the past month (61). Available clinical measures do not suggest wide-spread vitamin D deficiency in Canadian children/youth (60), however, the status of vitamin D in some sub-populations may warrant further consideration (e.g. adolescent females (14-18 years old) $(62,63)$. This group is an important focus considering obstetric complications for females later in life, as a result of distorted pelvis, bowing of the legs and dwarfing due to vitamin D and associated calcium deficiency $(64,65)$. Moreover, Canada's Food Guide $(66)$ was recently updated, with the "Milk and Alternatives" and "Meat and Alternatives" food groups now replaced with a "Protein Foods" group. Given these changes, it may be important to monitor both calcium and vitamin D intakes of children and adolescents in the coming years.

The largest prevalence of inadequate intakes of essential nutrients, such as vitamin B6, vitamin B12, folate, zinc and iron, were seen for adolescent girls $14-18$ years. These nutrients are of particular importance for adolescent females as the commencement of menstruation during this age group demands for increased nutritional needs. Similar results were reported previously for female adolescents in the US population (67). Environmental and social factors (e.g. dieting, body image concern) can lead to disordered eating behaviours (68), possibly increasing the risk of inadequate intakes of essential nutrients within this specific population group. A study of Canadian adolescents found that $4.5 \%$ of females met the criteria for an eating disorder (69). Considering these findings, adolescent females may be a particularly vulnerable for malnutrition; highlighting the importance of understanding the intake practices of this group to promote better health and nutrition.

Although sodium intakes were lower in this study compared to 2004 (70), Canadian children and adolescents are still consuming too much sodium in comparison to the CDRR. This is a major concern as recent Canadian data indicates that $7 \%$ of Canadian children and adolescents now have either borderline or overt hypertension (49). Additionally, emerging evidence indicates an association between high sodium intake and being obese and risk of developing 
cardiovascular disease in children and youth (71-73). Moreover, these high intakes of sodium may be a specific cause for concern for certain sub-populations of children and adolescents. For example, research has shown that children born with low birth weight may be at a higher risk for developing hypertension if they consume excess sodium across the lifespan (74). Due to an increased risk of cardiovascular disease associated with high sodium intakes, populationlevel sodium reduction interventions, such as the reformulation of specific food products, have been recommended (23) and have resulted in effectively decreasing sodium levels across the food supply chain in Canada (75).

The Canadian government has initiated several policies since 2015 to improve the nutritional outcomes of children and adolescents. As part of Health Canada's Healthy Eating Strategy, specific recommendations are being considered for food products specifically designed for children and youth (76). As an example, nutrition labelling regulations have been updated to include the percentage daily value of sodium on the Nutrition Facts table on packaged foods marketed to children aged 1-4 years old (77). A promising policy initiative to restrict marketing of unhealthy foods and beverages via traditional and digital marketing advertising (e.g. television advertising) to children under the age of 13 years is also being considered (77). Introduction of such policies may be particularly impactful in reducing the consumption of processed foods in childhood and to prevent NCDs in adulthood. Findings from the current study may act as a benchmark to monitor progress in the quality of Canadian children and adolescents' dietary intakes given the government's proposed policy changes.

\section{Strengths and Limitations}

This is the first study to utilize nationally representative data from 2015 CCHS - Nutrition to estimate nutrient intakes and the prevalence of nutrient inadequacy among Canadian children and adolescents, in addition to providing interpretation of the results and implications of these findings. Using both dietary recalls and applying the $\mathrm{NCl}$ method strengthen the results of these findings as most research in this field utilize only a single day of dietary recall, which is inappropriate to estimate usual intakes. Additionally, methodological considerations were incorporated in this study to strengthen the results, such as a robust outlier removal method recommended by Health Canada (36), accounting for misreporting bias and correcting for self-reported BMI (the published literature on CCHS 2004 do not account for either) and adjustments for covariates such as age, sex, weekend/weekday and sequence of dietary recall.

The results from the present study estimates nutrient intakes from food and beverage consumption only, i.e., the results do not consider supplement use. Furthermore, clinical measures of deficiency were not investigated as $2015 \mathrm{CCHS}$ - Nutrition does not provide these measures. Finally, this study relies on estimating nutrient intakes from foods and beverages found in the most recent national food composition database (the Canadian Nutrient File; CNF) (78). The CNF may not be updated for all nutrients and/or products (30); therefore, reported nutrient estimates reported herein are only as accurate as CNF 2015. Additionally, caution should be exercised when making comparisons between published results on 2004 and 2015 CCHS-Nutrition due to methodological differences in data collection, data processing and data analysis between the two surveys (28). Some of the methodological differences include differences in sample size and response rates, updates to the nutrient databases, use of usual intake estimation methods (e.g., Software for Intake Distribution Estimation (SIDE) vs. $\mathrm{NCl}$ ), handling of outliers and adjustment for certain covariates in estimation models. Further details on the differences between the two survey cycles can be found elsewhere (28).

\section{Conclusions}

Childhood and adolescence are important stages of human development with pronounced physical growth, reproductive maturation and cognitive transformation. The current study presents less-than-recommended intakes of some essential nutrient among Canadian children and adolescents; this indicates an urgent public health importance to improve the nutrient and diet quality of children and adolescents in Canada. Childhood and adolescent malnutrition presents a significant burden to health, and negatively impacts economic development and prosperity; the importance of adequate nutrition in children, especially among food insecure or marginalized communities, is unequivocal. These results provide insights into specific nutrients for target age-sex groups that may be an important foci for public health interventions aimed at improving diet quality and nutrient adequacy in Canada.

\section{Abbreviations}

non-communicable diseases (NCDs); Canadian Community Health Survey (CCHS); Dietary Reference Intakes (DRI); Public Use Microdata File (PUMF); National Cancer Institute (NCI); United States Department of Agriculture (USDA); Canadian Nutrient File (CNF); energy intakes (EI); total energy expenditure (TEE); Body Mass Index (BMI); World Health Organization (WHO); Estimated Average Requirement (EAR); balanced repeated replication (BRR); standard errors (SEs); Acceptable Macronutrient; Distribution Range (AMDR); Tolerable Upper Intake Level (UL);Adequate Intake (Al); Chronic Disease Risk Reduction (CDRR); National Health and Nutrition Examination Survey (NHANES); Software for Intake Distribution Estimation (SIDE)

\section{Declarations}

Ethics disclosure: This study was a secondary analyses of CCHS Nutrition - 2015 data, which is a voluntary health and nutrition survey. All information was de-identified and vetted by a trained member of Statistics Canada prior to release from the Toronto Research Data Center. Data for this study were collected under the authority of the Statistics Act of Canada. All procedures performed in studies involving human participants were in accordance with the ethical standards of the institutional and/or national research committee and with the 1964 Helsinki declaration and its later amendments or comparable ethical standards and were collected under the authority of the Statistics Act of Canada.

Consent for publication:Not Applicable 
Data availability: Data are publicly and freely available without restriction at Statistics Canada, https://www150.statcan.gc.ca/n1/pub/11-625-x/11-625x2010000-eng.htm.

Competing interests: MA held a Mitacs Elevate Postdoctoral Fellowship from September 2017 to September 2019, which is jointly funded by the Government of Canada Mitacs Program and the Nestlé Research Centre; however, neither of the organizations had a role in this research. The remaining authors declare no conflicts of interest.

Funding: Support for this research was obtained from Earle W. McHenry Research Chair Award (M.L.), Centre for Child Nutrition and Health Grant (\#002/2017/471141) and CIHR Project Grant (\#152179).

Author contributions: A.P.N, M.A. and M.R.L conceptualized and designed the study; A.P.N developed the coding for the analysis; A.P.N and M.A. conducted the statistical analyses, drafted and revised the manuscript; M.R.L reviewed the manuscript. All authors read and approved the final manuscript.

Acknowledgments: The authors would like to acknowledge the assistance of Mahsa Jessri, PhD (Assistant Professor, University of British Columbia) for assistance with the estimates of energy intake.

\section{References}

1. Statistics Canada. Table 13-10-0794-01: Measured adult body mass index (BMI) (World Health Organization classification), by age group and sex, Canada and provinces, Canadian Community Health Survey - Nutrition 2017 [Available from: https://www150.statcan.gc.ca/t1/tbl1/en/tv.action? pid $=1310079401$ \& pickMembers\%5B0\%5D = 1.1 \&pickMembers\%5B1\%5D =2.1\&pickMembers $\% 5 B 2 \% 5 D=3.1 \&$ pickMembers\%5B3\%5D=5.5 .

2. Government of Canada. Childhood Obesity 2019 [Available from: https://www.canada.ca/en/public-health/services/childhood-obesity/childhoodobesity.html.

3. Perrin JM, Bloom SR, Gortmaker SL. The increase of childhood chronic conditions in the United States. JAMA. 2007;297(24):2755-9.

4. Miller GF, Coffield E, Leroy Z, Wallin R. Prevalence and Costs of Five Chronic Conditions in Children. J Sch Nurs. 2016;32(5):357-64.

5. World Health Organization. Healthy Diet 2020 [Available from: https://www.who.int/news-room/fact-sheets/detail/healthy-diet.

6. World Health Organization. Global status report on noncommunicable diseases 2014. Geneva: World Health Organization; 2014.280 p. p.

7. World Health Organization. Global strategy on diet, physical activity and health. France2004 [Available from:

https://www.who.int/dietphysicalactivity/strategy/eb11344/strategy_english_web.pdf.

8. Smolin LA, Grosvenor MB, Gurfinkel D. Nutrition: Science and Applications. Third Canadian edition. ed2020. xxxiv, 764, 47, 10, 26 pages p.

9. Jessi M, Nishi SK, L'Abbe MR. Assessing the nutritional quality of diets of Canadian children and adolescents using the 2014 Health Canada Surveillance Tool Tier System. BMC Public Health. 2016;16:381.

10. Hack S, Jessri M, L'Abbe MR. Evaluating Diet Quality of Canadian Adults Using Health Canada's Surveillance Tool Tier System: Findings from the 2015 Canadian Community Health Survey-Nutrition. Nutrients. 2020;12(4).

11. Colapinto CKG, J.; St-Pierre, S.,. Trends and correlates of frequency of fruit and vegetable consumption, 2007 to 2014 . In: Canada H, editor. Ottawa2018.

12. Garriguet D. Diet quality in Canada. Health reports. 2009;20(3):41-52.

13. Garriguet D. Canadians' eating habits. Health reports. 2007;18(2):17-32.

14. Nshimyumukiza L, Lieffers JRL, Ekwaru JP, Ohinmaa A, Veugelers PJ. Temporal changes in diet quality and the associated economic burden in Canada. PLoS One. 2018;13(11):e0206877.

15. Signal LN, Stanley J, Smith M, Barr MB, Chambers TJ, Zhou J, et al. Children's everyday exposure to food marketing: an objective analysis using wearable cameras. Int J Behav Nutr Phys Act. 2017;14(1):137.

16. Vergeer L, Vanderlee L, Potvin Kent M, Mulligan C, L'Abbe MR. The effectiveness of voluntary policies and commitments in restricting unhealthy food marketing to Canadian children on food company websites. Appl Physiol Nutr Metab. 2019;44(1):74-82.

17. Health Canada. Do Canadian children meet their nutrient requirements through food intake alone? [Electronic monograph in PDF format]. [Ottawa]: Health Canada; 2009. Available from: http://resource.library.utoronto.ca/eir/EIRdetail.cfm?Resources_ID=1572251

18. http://resource.library.utoronto.ca/eir/EIRdetail.cfm?Resources_ID=1572252.

19. Health Canada and Statistics Canada. Canadian Community Health Survey, Cycle 2.2, Nutrition (2004) - Nutrient Intakes from Food, Volume 1-3: Provincial, Regional and National Summary Data Tables. Ottawa; 2009.

20. Statistics Canada. Canadian Community Health Survey - Nutrition: Nutrient intakes from food and nutritional supplements; Available in CANSIM: tables 105-2017 to 105-2022, 105-2027 and 105-2028. In: Canada S, editor. 2017.

21. Health Canada. Sodium Intake of Canadians in 20172017 [Available from: https://www.canada.ca/en/health-canada/services/publications/foodnutrition/sodium-intake-canadians-2017.html\#a9.

22. Statistics Canada. Canadian Community Health Survey - Nutrition: Nutrient intakes from food and nutritional supplements; Available in CANSIM: tables 105-2017 to 105-2022, 105-2027 and 105-2028. 2017 [Available from: https://www150.statcan.gc.ca/n1/daily-quotidien/170620/dq170620b-cansimeng.htm.

23. Institute of Medicine. Dietary Reference Intakes 1995-2019 [Available from: https://www.canada.ca/en/health-canada/services/food-nutrition/healthyeating/dietary-reference-intakes.html. 
24. National Academies of Sciences Engineering and Medicine. Dietary Reference Intakes for Sodium and Potassium. Oria M, Harrison M, Stallings VA, editors. Washington (DC)2019.

25. Institute of Medicine (US) Panel on Micronutrients., Panel on Micronutrients Subcommittees on Upper Reference Levels of Nutrients and of Interpretation and Use of Dietary Reference Intakes and the Standing Committee on the Scientific Evaluation of Die. Dietary Reference Intakes for Vitamin A, Vitamin K, Arsenic, Boron, Chromium, Copper, lodine, Iron, Manganese, Molybdenum, Nickel, Silicon, Vanadium, and Zinc. [S.I.]: National Academies Press (US); 2001. Available from: http://www.nap.edu/openbook.php?record_id=10026

26. http://www.ncbi.nlm.nih.gov/books/NBK222310.

27. Statistics Canada. Canadian Community Health Survey - Nutrition: Public Use Microdata File 2019 [Available from: https://www150.statcan.gc.ca/n1/en/catalogue/82M0024X.

28. National Cancer Institute. Usual Dietary Intake: SAS Macros for the NCI Method 2018 [Available from: https://epi.grants.cancer.gov/diet/usualintakes/macros.html.

29. Health Canada. 2015 Canadian Community Health Survey - Nutrition 2015 [Available from: https://www.canada.ca/en/health-canada/services/foodnutrition/food-nutrition-surveillance/health-nutrition-surveys/canadian-community-health-survey-cchs/2015-canadian-community-health-survey-nutritionfood-nutrition-surveillance.html.

30. Health Canada. 2015 Canadian Community Health Survey - Reference Guide to Understanding and Using the Data 2017 [Available from: http://sda.chass.utoronto.ca/sdaweb/dli2/cchs/cchs2015_nu/more_doc/CCHS_NU_2015_PUMF_User_Guide.pdf; https://www.canada.ca/en/healthcanada/services/food-nutrition/food-nutrition-surveillance/health-nutrition-surveys/canadian-community-health-survey-cchs/reference-guideunderstanding-using-data-2015.html\#a4.2.

31. Moshfegh AJ, Rhodes DG, Baer DJ, Murayi T, Clemens JC, Rumpler WV, et al. The US Department of Agriculture Automated Multiple-Pass Method reduces bias in the collection of energy intakes. Am J Clin Nutr. 2008;88(2):324-32.

32. Health Canada. Canadian Nutrient File 2015 [Available from: https://food-nutrition.canada.ca/cnf-fce/index-eng.jsp.

33. Health Canada. 2015 Canadian Community Health Survey - Nutrition User Guide 2018 [Available from: http://sda.chass.utoronto.ca/sdaweb/dli2/cchs/cchs2015_nu/more_doc/CCHS_NU_2015_PUMF_User_Guide.pdf.

34. Garriguet D. Accounting for misreporting when comparing energy intake across time in Canada 2018 [Available from: https://www150.statcan.gc.ca/n1/en/pub/82-003-x/2018005/article/54965-eng.pdf.

35. Institute of Medicine (U.S.). Panel on Macronutrients., Institute of Medicine (U.S.). Standing Committee on the Scientific Evaluation of Dietary Reference Intakes. Dietary reference intakes for energy, carbohydrate, fiber, fat, fatty acids, cholesterol, protein, and amino acids. Washington, D.C.: National Academies Press; 2005. xxv, 1-972, 1259-331 p. p.

36. Cole TJ, Bellizzi MC, Flegal KM, Dietz WH. Establishing a standard definition for child overweight and obesity worldwide: international survey. BMJ. 2000;320(7244):1240-3.

37. U.S. Department of Health and Human Services. Energy levels used for assignment of individuals to USDA Food Patterns 2019 [Available from: https://health.gov/our-work/food-nutrition/2015-2020-dietary-guidelines/advisory-report/appendix-e-3/appendix-e-31a3.

38. Davis KA, Gonzalez A, Loukine L, Qiao C, Sadeghpour A, Vigneault M, et al. Early Experience Analyzing Dietary Intake Data from the Canadian Community Health Survey-Nutrition Using the National Cancer Institute (NCl) Method. Nutrients. 2019;11(8).

39. Tooze JA, Kipnis V, Buckman DW, Carroll RJ, Freedman LS, Guenther PM, et al. A mixed-effects model approach for estimating the distribution of usual intake of nutrients: the NCl method. Stat Med. 2010;29(27):2857-68.

40. Tooze JA, Midthune D, Dodd KW, Freedman LS, Krebs-Smith SM, Subar AF, et al. A new statistical method for estimating the usual intake of episodically consumed foods with application to their distribution. J Am Diet Assoc. 2006;106(10):1575-87.

41. Institute of Medicine (U.S.). Subcommittee on Interpretation and Uses of Dietary Reference Intakes., Institute of Medicine (U.S.). Subcommittee on Upper Reference Levels of Nutrients., Institute of Medicine (U.S.). Standing Committee on the Scientific Evaluation of Dietary Reference Intakes. Dietary reference intakes. Applications in dietary assessment: a report of the Subcommittees on Interpretation and Uses of Dietary Reference Intakes and the Standing Committee on the Scientific Evaluation of Dietary Reference Intakes, Food and Nutrition Board, Institute of Medicine. Washington, D.C.: National Academy Press; 2000. xvii, 287 p. p.

42. Ross AC, Institute of Medicine (U. S.). Committee to Review Dietary Reference Intakes for Vitamin D and Calcium. Dietary reference intakes calcium, vitamin D. Washington, DC: National Academies Press; 2011. Available from: http://www.nap.edu/openbook.php?record_id=13050

43. http://www.ncbi.nlm.nih.gov/books/NBK56070.

44. Katamay SW, Esslinger KA, Vigneault M, Johnston JL, Junkins BA, Robbins LG, et al. Eating well with Canada's Food Guide (2007): development of the food intake pattern. Nutr Rev. 2007;65(4):155-66.

45. Health Canada. Do Canadian adults meet their nutrient requirements through food intake alone? [Electronic monograph in PDF format]. [Ottawa]: Health Canada; 2012. Available from: http://resource.library.utoronto.ca/eir/EIRdetail.cfm?Resources_ID=1579433.

46. Beaton G. Beaton's Full Probability Method for Iron 2015 [Available from: https://www.abs.gov.au/AUSSTATS/abs@.nsf/Lookup/4363.0.55.001Chapter6510312011-13.

47. Health Canada and Statistics Canada. Nutrient Intakes from Food: Provincial, Regional and National Summary Data Tables Volume 2. In: Canada H, editor. Ottawa: Ministry of Health; 2000.

48. Health Canada. Do Canadian adolescents meet their nutrient requirements through food intake alone? [Electronic monograph in PDF format]. Ottawa: Health Canada; 2009. Available from: http://resource.library.utoronto.ca/eir/EIRdetail.cfm?Resources_ID=1572243

Page $11 / 14$ 
49. http://resource.library.utoronto.ca/eir/EIRdetail.cfm?Resources_ID=1572245.

50. Greer FR, Krebs NF, American Academy of Pediatrics Committee on N. Optimizing bone health and calcium intakes of infants, children, and adolescents. Pediatrics. 2006;117(2):578-85.

51. Dietary Guidelines Advisory Committee Members. Shifts Needed To Align With Healthy Eating Patterns: U.S Department of Health and Human Services; U.S. Department of Agriculture 2015 [Available from: https://health.gov/our-work/food-nutrition/2015-2020-dietary-guidelines/guidelines/chapter-2/.

52. Health Canada. Usual Intakes from Food for Energy, Nutrients and Other Dietary Components (2004 and 2015 CCHS-Nutrition) derived from Statistics Canada's 2004 and 2015 Canadian Community Health Survey, Nutrition, Share file Ottawa2019 [Available from: https://open.canada.ca/data/en/dataset/31599960-2c1e-4d90-a9d9-979ad0e1abb4.

53. Statistics Canada. Health Fact Sheets: Blood Pressure of Children and Youth, 2012-2015. 2016 [Available from: https://www150.statcan.gc.ca/n1/pub/82-625-x/2016001/article/14659-eng.htm.

54. Moubarac JC, Batal M, Martins AP, Claro R, Levy RB, Cannon G, et al. Processed and ultra-processed food products: consumption trends in Canada from 1938 to 2011. Can J Diet Pract Res. 2014;75(1):15-21.

55. Polsky JY, Moubarac JC, Garriguet D. Consumption of ultra-processed foods in Canada. Health Rep. 2020;31(11):3-15.

56. Statistics Canada. Fruit and vegetable consumption 2017 [Available from: https://www150.statcan.gc.ca/n1/pub/82-625-x/2019001/article/00004eng.htm.

57. Salamoun MM, Kizirian AS, Tannous RI, Nabulsi MM, Choucair MK, Deeb ME, et al. Low calcium and vitamin D intake in healthy children and adolescents and their correlates. Eur J Clin Nutr. 2005;59(2):177-84.

58. Rampersaud GC, Bailey LB, Kauwell GP. National survey beverage consumption data for children and adolescents indicate the need to encourage a shift toward more nutritive beverages. J Am Diet Assoc. 2003;103(1):97-100.

59. American Academy of Pediatrics. The use and misuse of fruit juices in pediatrics. Pediatrics. 2001;107:1210-3.

60. Frary CD, Johnson RK, Wang MQ. Children and adolescents' choices of foods and beverages high in added sugars are associated with intakes of key nutrients and food groups. J Adolesc Health. 2004;34(1):56-63.

61. Garriguet D. Beverage consumption of children and teens. Health reports. 2008;19(4):17-22.

62. Tugault-Lafleur CN, Black JL. Differences in the Quantity and Types of Foods and Beverages Consumed by Canadians between 2004 and 2015 . Nutrients. 2019;11(3).

63. Statista. Consumption of milk per capita in Canada from 2004 to 20182020 [Available from: https://www.statista.com/statistics/438584/consumptionof-milk-per-capita-canada/.

64. Statistics Canada. Health at a Glance: Vitamin D blood levels of Canadians 2015 [Available from: https://www150.statcan.gc.ca/n1/pub/82-624x/2013001/article/11727-eng.htm.

65. Health Canada. Map of Past Month Consumption of Vitamin and Mineral Supplements in Children in Canada 2008 [Available from: https://www.canada.ca/en/health-canada/services/food-nutrition/food-nutrition-surveillance/canada-nutrition-atlas/maps-indicator/map-past-monthconsumption-vitamin-mineral-supplements-children-canada-both-males-females.html.

66. Moore C, Murphy MM, Keast DR, Holick MF. Vitamin D intake in the United States. J Am Diet Assoc. 2004;104(6):980-3.

67. McCarthy D, Collins A, O'Brien M, Lamberg-Allardt C, Jakobsen J, Charzewska J, et al. Vitamin D intake and status in Irish elderly women and adolescent girls. Ir J Med Sci. 2006;175(2):14-20.

68. Molgaard C, Michaelsen KF. Vitamin D and bone health in early life. Proc Nutr Soc. 2003;62(4):823-8.

69. Cashman KD. Vitamin D in childhood and adolescence. Postgrad Med J. 2007;83(978):230-5.

70. Health Canada. Canada's Food Guide 2019 [Available from: https://food-guide.canada.ca/en/.

71. Moore LL, Singer MR, Qureshi MM, Bradlee ML, Daniels SR. Food group intake and micronutrient adequacy in adolescent girls. Nutrients. 2012;4(11):1692-708.

72. Ciao AC, Loth K, Neumark-Sztainer D. Preventing eating disorder pathology: common and unique features of successful eating disorders prevention programs. Curr Psychiatry Rep. 2014;16(7):453.

73. Flament MF, Henderson K, Buchholz A, Obeid N, Nguyen HN, Birmingham M, et al. Weight Status and DSM-5 Diagnoses of Eating Disorders in Adolescents From the Community. J Am Acad Child Adolesc Psychiatry. 2015;54(5):403-11 e2.

74. Garriguet D. Sodium consumption at all ages. Health Rep. 2007;18(2):47-52.

75. Grimes CA, Riddell LJ, Campbell KJ, He FJ, Nowson CA. 24-h urinary sodium excretion is associated with obesity in a cross-sectional sample of Australian schoolchildren. Br J Nutr. 2016;115(6):1071-9.

76. Ma Y, He FJ, MacGregor GA. High salt intake: independent risk factor for obesity? Hypertension. 2015;66(4):843-9.

77. Lee SK, Kim MK. Relationship of sodium intake with obesity among Korean children and adolescents: Korea National Health and Nutrition Examination Survey. Br J Nutr. 2016;115(5):834-41.

78. Simonetti GD, Raio L, Surbek D, Nelle M, Frey FJ, Mohaupt MG. Salt sensitivity of children with low birth weight. Hypertension. 2008;52(4):625-30.

79. Arcand J, Jefferson K, Schermel A, Shah F, Trang S, Kutlesa D, et al. Examination of food industry progress in reducing the sodium content of packaged foods in Canada: 2010 to 2013. Appl Physiol Nutr Metab. 2016;41(6):684-90.

80. Health Canada. Healthy Eating Strategy: Toward Front-of-Package Nutrition Labels for Canadians: Consultation Document Canada2016 [Available from: https://www.canada.ca/en/services/health/campaigns/vision-healthy-canada/healthy-eating.html.

Page $12 / 14$ 
81. Health Canada. Canada Gazette Part II: Regulations Amending the Food and Drug Regulations (Nutrition Labelling, Nutrient Content Claims and Health Claims). 2003 [Available from: http://canadagazette.gc.ca/partll/2003/20030101/pdf/g2-13701.pdf.

82. Health Canada. Search online for foods using the Canadian Nutrient File 2010 [Available from: https://www.canada.ca/en/health-canada/services/foodnutrition/healthy-eating/nutrient-data.html.

\section{Figures}
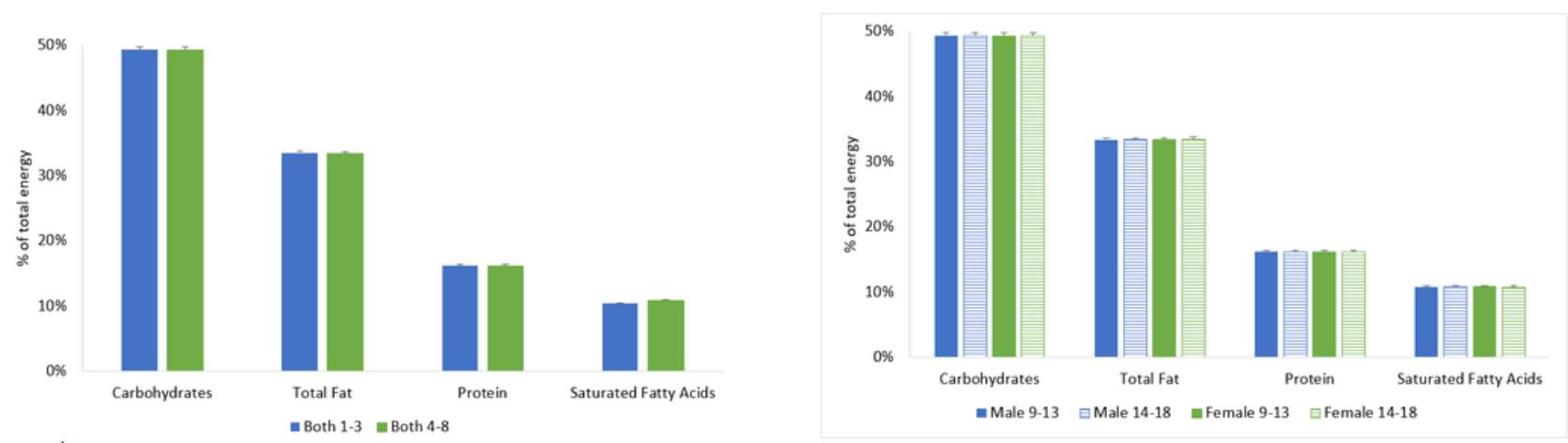

A

$\mathrm{B}$

Figure 1

a. Percentage of total energy from macronutrients, Canadian children (2-8y). Data Source: Statistics Canada, Canadian Community Health Survey, Nutrition (2015) - Public Use Microdata File. All intakes are based on food and beverage consumption only and exclude intakes from vitamin or mineral supplements. The National Cancer Institute Method (NCl Method) for estimating usual dietary intake was used. The following covariates were adjusted for in all NCI models: age, sex, dietary misreporting status, day of the week (weekend vs. weekday), and sequence of dietary recall analysed (first or second). Outliers for nutrient intake were defined and removed using the methodology reported in Davis et al. (36). The number of respondents removed due to outlier methodology varied between 1-5 respondents for each nutrient. The analytical sample size before outlier removal was $n=5,493$.. All estimates were weighted for population-level estimates using sampling survey weights provided by Statistics Canada. All reported SEs were bootstrapped using the 500 boot weights provided by Statistics Canada. The SE represents the more conservative (i.e., larger) standard error estimate from the NCl output representing the proportion of the population meeting either the upper or lower AMDR bound.

b. Percentage of total energy from macronutrients, Canadian adolescents (9-18y). Data Source: Statistics Canada, Canadian Community Health Survey, Nutrition (2015) - Public Use Microdata File. All intakes are based on food and beverage consumption only and exclude intakes from vitamin or mineral supplements. The National Cancer Institute Method (NCl Method) for estimating usual dietary intake was used. The following covariates were adjusted for in all NCl models: age, sex, dietary misreporting status, day of the week (weekend vs. weekday), and sequence of dietary recall analysed (first or second). Outliers for nutrient intake were defined and removed using the methodology reported in Davis et al. (36). The number of respondents removed due to outlier methodology varied between 1-5 respondents for each nutrient. The analytical sample size before outlier removal was $n=5,493 .$. All estimates were weighted for population-level estimates using sampling survey weights provided by Statistics Canada. All reported SEs were bootstrapped using the 500 boot weights provided by Statistics Canada. The SE represents the more conservative (i.e., larger) standard error estimate from the $\mathrm{NCl}$ output representing the proportion of the population meeting either the upper or lower AMDR bound. 


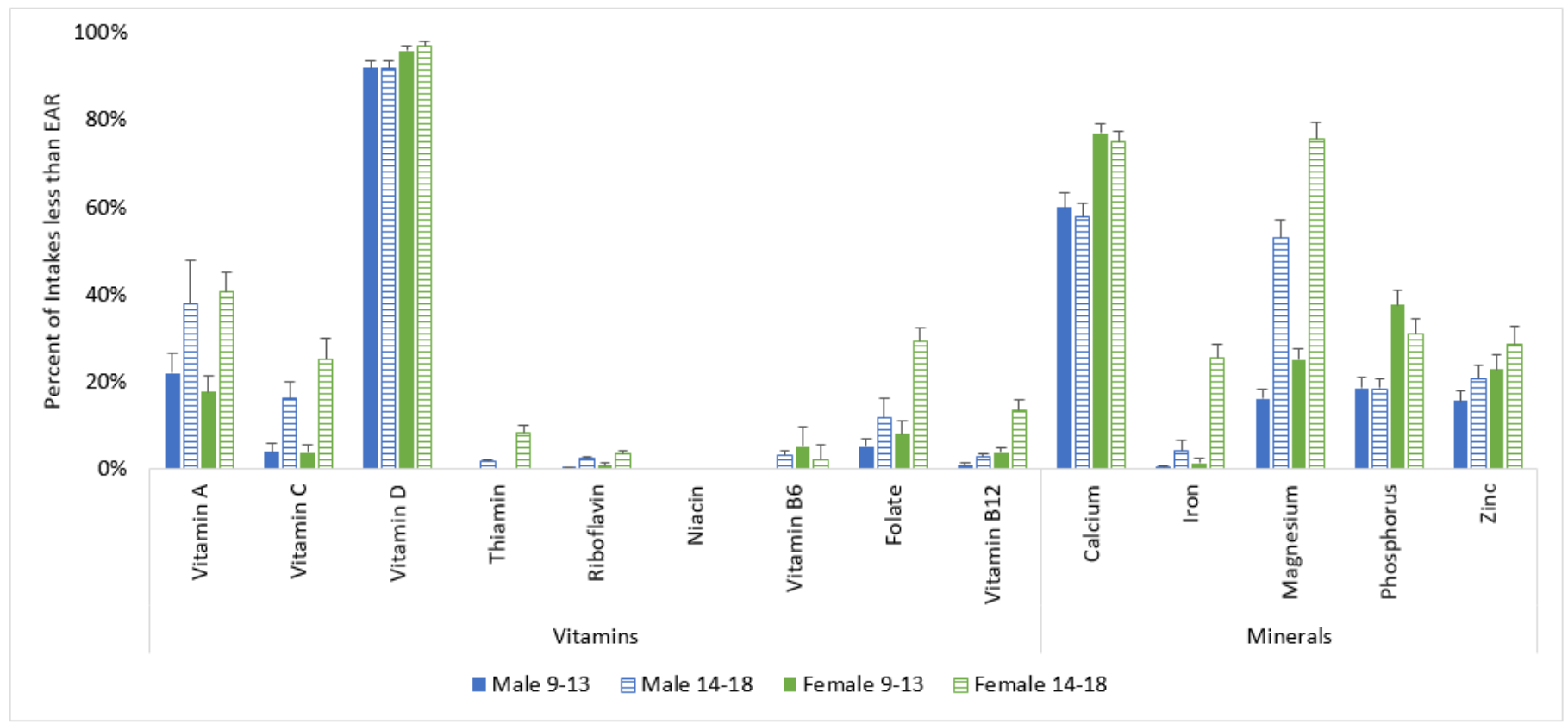

Figure 2

Prevalence of inadequate intakes for nutrients with an Estimated Energy Requirement, Canadian adolescents (9-18y). Data Source: Statistics Canada, Canadian Community Health Survey, Nutrition (2015) - Public Use Microdata File. All intakes are based on food and beverage consumption only and exclude intakes from vitamin or mineral supplements. The National Cancer Institute Method (NCI Method) for estimating usual dietary intake was used. The following covariates were adjusted for in all $\mathrm{NCl}$ models: age, sex, dietary misreporting status, day of the week (weekend vs. weekday), and sequence of dietary recall analysed (first or second). Outliers for nutrient intake were defined and removed using the methodology reported in Davis et al. (36). The number of respondents removed due to outlier methodology varied between 1-5 respondents for each nutrient. The analytical sample size before outlier removal was $\mathrm{n}=5,493$.. All estimates were weighted for population-level estimates using sampling survey weights provided by Statistics Canada. All reported SEs were bootstrapped using the 500 boot weights provided by Statistics Canada. Data not shown in a graphical representation for children $2-8 \mathrm{y}$ as the prevalence of inadequate intakes was very low for majority of the nutrients (exception vitamin $D$ and calcium).

\section{Supplementary Files}

This is a list of supplementary files associated with this preprint. Click to download.

- ChildrenNutrientIntakes20211203SUPPLMATERIALS.docx 